Gerber, Markus, Best, Simon,

Meerstetter, Fabienne, Walter, Marco, Ludyga, Sebastian, Brand, Serge, Bianchi, Renzo, Madigan, Daniel J. ORCID:

https://orcid.org/0000-0002-9937-1818, Isoard-Gautheur, Sandrine and Gustafsson, Henrik (2018) Effects of stress and mental toughness on burnout and depressive symptoms: A prospective study with young elite athletes. Journal of Science and Medicine in Sport, 21 (12). pp. 1200-1205.

Downloaded from: http://ray.yorksj.ac.uk/id/eprint/3101/

The version presented here may differ from the published version or version of record. If you intend to cite from the work you are advised to consult the publisher's version: https://www.sciencedirect.com/science/article/pii/S1440244018301701

Research at York St John (RaY) is an institutional repository. It supports the principles of open access by making the research outputs of the University available in digital form. Copyright of the items stored in RaY reside with the authors and/or other copyright owners. Users may access full text items free of charge, and may download a copy for private study or non-commercial research. For further reuse terms, see licence terms governing individual outputs. Institutional Repository Policy Statement

\title{
RaY
}

Research at the University of York St John

For more information please contact RaY at ray@yorksj.ac.uk 


\section{Effects of stress and mental toughness on burnout and depressive symptoms: A prospective}

study with young elite athletes

Markus Gerber ${ }^{1}$, Simon Best $^{1}$, Fabienne Meerstetter ${ }^{1}$, Marco Walter $^{1}$, Sebastian Ludyga ${ }^{1}$, Serge Brand $^{1,2,3}$, Renzo Bianchi ${ }^{4}$, Daniel J. Madigan ${ }^{5}$, Sandrine Isoard-Gautheur ${ }^{6}$, Henrik Gustafsson ${ }^{7}$

${ }^{1}$ Department of Sport, Exercise and Health, University of Basel, Basel, Switzerland

${ }^{2}$ Psychiatric Clinics (UPK), Center for Affective, Stress and Sleep Disorders, University of Basel, Basel, Switzerland

${ }^{4}$ Kermanshah University of Medical Sciences (KUMS), Psychiatry Department, Substance Abuse Prevention Center and Sleep Disorders Research Center, Kermanshah, Iran

${ }^{4}$ Institut of Work and Organizational Psychology, University of Neuchâtel, Neuchâtel, S witzerland ${ }^{5}$ School of Sport, York St. John University, York, United Kingdom

${ }^{6}$ Laboratoire Sport et Environnement Social, Université Grenoble Alpes, Grenoble Cedex, France ${ }^{7}$ Department of Health Sciences, Karlstad University, Karlstad, Sweden

\section{Corresponding author:}

Prof. Dr. Markus Gerber, Department of Sport, Exercise and Health, University of Basel, St. JakobTurm, Birsstrasse 320B, CH-Basel, 0041612074783 (phone), 0041612074789 (fax), markus.gerber@unibas.ch (email)

\section{Abstract}

Objectives: To examine in a sample of young elite athletes (a) the presence of clinically relevant symptoms of burnout and depression, and (b) a possible interaction of perceived stress and mental toughness in the prediction of burnout and depressive symptoms.

Design: 6-month prospective study. 
Methods: A representative sample of 257 young elite athletes $(M=16.82$ years, $S D=1.44,36 \%$ females) was recruited in North-Western Switzerland. 197 athletes were followed-up across a 6-month period. Burnout was assessed with the Shirom-Melamed Burnout Measure (SMBM), and depression with the 9-item depression module of the Patient Health Questionnaire (PHQ). Values of $\geq 4.40$ $(\mathrm{SMBM})$ and >14 (PHQ-9) were considered indicative of clinically relevant burnout or depression. Stress perceptions were assessed with the Perceived Stress Scale (PSS), and mental toughness with the Mental Toughness Questionnaire (MTQ). Hierarchical regression analyses were used to test stressbuffering effects.

Results: The percentage of athletes with clinically relevant levels of burnout and depressive symptoms was $12 \%$ and $9 \%$, respectively. Both cross-sectional and prospective analyses showed that compared to participants with low mental toughness, those with higher mental toughness scores reported significantly fewer mental health issues, when exposed to high stress. By contrast, when stress levels were low, mental toughness was unrelated to psychological health complaints.

Conclusions: About every tenth young elite athlete reported burnout or depressive symptoms of potential clinical relevance. While high perceived stress was associated with increased psychological health complaints, mental toughness was able to off-set some of the negative consequences resulting from high stress exposure.

Key words: Burnout; elite athletes; depressive symptoms; quality of life; stress

\section{Introduction}

In competitive sports, stress is a common phenomenon and elite athletes encounter a variety of different stressors. ${ }^{1}$ Scholars have argued that young elite athletes are at particular risk for high perceived stress levels due to high training loads, uncertainty associated with selection processes, role conflicts and biological changes occurring during adolescence, and high pressure to perform well both in sport and at school. ${ }^{2}$ Therefore, it is no surprise that psychological disorders are highly prevalent among elite athletes. ${ }^{3}$ For instance, in a study of Australian athletes, almost half of the participants (46\%) reported at least one mental health issue, including depression, eating disorder, general 
psychological distress, and anxiety disorders. ${ }^{4}$ As yet, however, prevalence rates for young elite athletes are unclear. Gaining a deeper understanding about mental health issues and possible protective factors among young athletes is important because such issues have been associated with dropout from elite sport. ${ }^{5}$

The cognitive-transactional model of stress states that when an individual is faced with harm, loss, threat or challenge several transactions occur between the person and the environment. These transactions refer to a balance of forces that environmental demands tax or exceed the resources of a person. If a person is unable to meet or neutralize such a demand, negative consequences may occur. However, such demands can also stem from internal pressures, and the consequences depend on multiple appraisal processes. ${ }^{6}$ In line with this stress model, previous studies have shown that individual appraisal processes impact on athletes' response to particular stressors. ${ }^{7}$ Hence, some athletes seem to be more resilient than others when they face stressful life circumstances. ${ }^{8,9}$ Following Luthar and Cicchetti, ${ }^{10}$ resilience is a dynamic process and describes the fact that individuals can show positive adaptation (e.g., do not develop symptoms of mental ill-health) despite exposure to risk and adversity. Generally, resilience researchers distinguish between vulnerability and protective factors. While the former describe factors that exacerbate the negative effects of risk circumstances, the latter contribute to a modification of the effects of risk in a favourable direction. ${ }^{10}$ In the domain of elite sport, Gustafsson et al. ${ }^{11}$ suggested that a number of factors can increase the risk of athletes to develop burnout symptoms such as physical fatigue, cognitive weariness and emotional exhaustion. ${ }^{12}$ Such risk factors are perfectionistic concerns, elevated trait anxiety, low social support, low autonomy, lack of coping skills, unfavourable goal orientation, exposure to a negative motivational climate, or a unidimensional athlete identity. ${ }^{13}$ However, it is noteworthy that Gustafsson et al.'s integrated model does not comprise protective factors, although previous research found that both personal and social factors can protect against negative psychosocial outcomes among elite athletes. ${ }^{14} \mathrm{With}$ regard to personal factors, Madigan and Nicholls recently showed among junior athletes that high mental toughness predicted residual decreases in burnout symptoms across a 3-month period, and thus offered some protection against burnout symptoms in sport. ${ }^{15}$ Although there are current debates about the 
way how to define mental toughness, Lin et al. ${ }^{16}$ recently defined mental toughness as a multidimensional personality construct, which helps people successfully cope with stressors and to strive when faced with challenging situations. Other researchers have highlighted that mental toughness reflects a set of positive psychological resources that may impact on the way people appraise and deal with demanding circumstances in order to achieve their goals. ${ }^{17}$ While Madigan and Nicholls ${ }^{15}$ assessed a direct link between mental toughness and burnout symptoms, from a resilience perspective, it would be interesting to know whether a mental toughness 'mind-set' (as a protective factor that facilitates resilience) is particularly helpful to prevent mental health issues when athletes are exposed to high stress levels. Evidence for a stress-buffering effect of mental toughness on mental health issues was reported previously, albeit outside the realm of competitive elite sport. For instance, Gerber and colleagues showed in high school students, vocational students, and university students that those with high mental toughness scores were less likely to report mental health issues, if exposed to stressful life circumstances. ${ }^{18-20}$ In their studies, mental toughness was defined via the four key components of control, commitment, challenge and confidence, as defined in the 4C model proposed by Clough and colleagues. ${ }^{21}$ While control describes the feeling of being influential in the face of the varied contingencies of life, commitment reflects individuals' tendency of becoming involved in experiences rather than experiencing alienation from whatever one encounters. Furthermore, challenge is the belief that change, rather than stability, is normal in life and that the anticipation of changes is an interesting incentive to growth rather than a threat to security. Finally, confidence describes how individuals feel valuable and competent in overcoming general and interpersonal problems.

Therefore, the main purpose of the present study was to examine (a) the presence of clinically relevant symptoms of burnout and depression among a sample of young elite athletes, and (b) a possible interaction of perceived stress and mental toughness on psychological well-being. In so doing, we investigated both cross-sectional and prospective relationships. Based on previous research with non-athletes, ${ }^{18-20}$ elite athletes with high levels of mental toughness were expected to show better psychological well-being at high levels of perceived stress than peers with lower levels of mental toughness. 


\section{Methods}

All students attending Swiss Olympic Sport Classes in the North-Western, German-speaking part of Switzerland were eligible for this prospective study. Adolescents need a Swiss Olympic Talents card to attend these classes, to certify that they belong to the top regional or national athletes in their sport and/or that they are part of a regional or national squad. Generally, these classes intend to facilitate the combination of school and elite sport (e.g., lower number of lessons per week, extended school duration). Participation was voluntary and all students were assured confidentiality. All participants provided informed written consent before the beginning of the data collection. Using the same instruments, data were collected in November-December 2016, and after a 6-month follow-up period in May-June 2017. All students responded to a written questionnaire, which was composed of psychological instruments with sound psychometric properties (see below). The study was approved by the local ethics committee (Ethical Review Board of Northern and Central Switzerland, Nr. 201601535b). A power analysis (using $\mathrm{G}^{*}$ Power 3.1) revealed that at least 208 participants are needed at follow-up to predict burnout symptoms with mental toughness across time (Slope H1 = -0.20, (alpha: 0.05, power: 0.80). Assuming a dropout rate of $20 \%$, the target sample size was estimated at 250 students at baseline.

In total, 257 athletes (163 males and 94 females; age: $M=16.82$ years, $S D=1.44$; range: $14-22$ years) took part in the baseline assessment. The athletes engaged in the following sports: Soccer ( $n=61)$, handball $(n=26)$, volleyball $(n=23)$, swimming $(n=19)$, judo $(n=17)$, track and field $(n=16)$, tennis $(n=15)$, uni hockey $(n=12)$, karate $(n=10)$, mountain bike $(n=7)$, golf $(n=5)$, others $(<5$ athletes; $n=46$ ). 197 athletes (125 males and 73 females; age: $M=16.83, S D=1.40$ ) completed the follow-up data assessment. Dropout analyses revealed that dropouts and students who participated in the follow-up assessment did not differ $(p>.05)$ with regard to any of the potential confounders or main study variables (see following paragraphs for more information about study variables and confounders).

Perceived stress during the past month was assessed with the widely used 10-item Perceived Stress Scale (PSS). ${ }^{22}$ The PSS is based on cognitive-transactional stress theory. ${ }^{6}$ Accordingly, 
participants were asked how often they find their lives to be overwhelming, uncontrollable, and unpredictable (e.g., "In the last month, how often have you felt that you were effectively coping with important changes that were occurring in your life?"). Answers were given on a 5-point Likert scale, ranging from 1 (never) to 5 (very often). Four items were reverse-poled and had to be recoded before calculating the sum score. Higher scores reflected higher levels of perceived stress. Evidence for the reliability and validity of this instrument has been provided previously. ${ }^{18}$ Information regarding factorial validity of the PSS in the present sample is provided as supplementary online material (as for all study variables). In the present sample, the Cronbach's alpha was satisfactory $(\alpha=.80)$.

Mental toughness was assessed with the 18-item short form of the MTQ48 ${ }^{21}$, which measures propensity to handle the demands of environmental stressors (e.g., "I don't usually give up under pressure"). The short form includes items from each dimension that constitute the MTQ48 (commitment, challenge, control, confidence). The short form scores are highly correlated $(r=.87$, $p<.001)$ with the MTQ48 in elite athletes, ${ }^{21}$ and previous studies supported the internal consistency of the MTQ18. ${ }^{18}$ Answers on the short form were given on a 5-point rating scale with anchors 1 (strongly disagree) and 5 (strongly agree). The mean was taken to obtain an overall score. In the present sample, the Cronbach's alpha was satisfactory $(\alpha=.75)$.

Burnout symptoms were assessed with the 14-item version of the Shirom-Melamed Burnout Measure (SMBM). ${ }^{23}$ The original SMBM consists of the three subscales labelled physical fatigue (six items: e.g., "I feel physically drained."), cognitive weariness (five items: e.g., "I feel I am not thinking clearly."), and emotional exhaustion (three items: e.g., "I feel I am unable to be sensitive to the needs of coworkers and customers."). The wording of the three items assessing emotional exhaustion was slightly changed to make the items more suitable for adolescents. Thus, instead of referring to coworkers and customers, we used a more open formulation, and referred to people in general. Response options range from 1 (never or almost never) to 7 (always or almost always), referring to experiences during the past month. The mean score is built to generate an overall index, with higher scores reflecting higher burnout symptoms. Following Lundgren-Nilsson et al., a score of $\geq 4.40$ 
indicates clinically relevant levels of burnout symptoms. ${ }^{24}$ In the present sample, the Cronbach's alpha was satisfactory $(\alpha=.92)$.

Depressive symptoms were assessed with the 9-item depression module of the Patient Health Questionnaire (PHQ-9). ${ }^{25}$ The PHQ-9 refers to the DSM-IV diagnosis criteria for major depressive disorder. Responses range from 0 (not at all) to 3 (nearly every day). Items are summed to obtain a general index, with higher scores reflecting higher symptomatology. The PHQ-9 can be used to assess severity of depressive symptoms, with scores of $>14$ reflecting clinically relevant depressive symptoms. ${ }^{25}$ In the present sample, the Cronbach's alpha was satisfactory $(\alpha=.85)$.

The following potential confounders were considered: Gender (male vs female), age, training load (including competitions), years participating in competitive sport, current injury (yes vs. no), and use of medication (yes vs. no).

To address our research questions, the following statistical analyses were performed: First, descriptive statistics ( $M, S D$, range, skewness, kurtosis) were calculated to describe the main study variables. Second, a series of analyses of variance (ANOVAs) and Pearson product moment correlations were calculated to examine how potential confounders were associated with stress, mental toughness and mental health outcomes. Third, Pearson product moment correlations were run to test bivariate relationships between the predictor (stress), moderator (mental toughness) and outcome variables (burnout symptoms, depressive symptoms). Hierarchical regression analyses were calculated to test whether perceived stress and mental toughness interact in the prediction of mental health outcomes. Interaction effects were tested based on both cross-sectional and prospective data. When we examined the prospective data, we first controlled for demographic and social background variables that showed a significant bivariate association with at least one of the outcome variables (Step 1). We then controlled for baseline levels of the dependent variable (Step 2). Next, we entered the baseline predictors of perceived stress (Step 3), followed by mental toughness (Step 4). Finally, the interaction between perceived stress and mental toughness was examined (Step 5). The same procedure was used in the cross-sectional analyses (but Step 2 was not included). Stress and mental toughness were centered before the interaction term was calculated (by multiplying stress with mental toughness). The 
following statistical coefficients are shown in the results section: (i) the multiple correlation coefficient squared $R^{2}$ for the entire model after the final step, (ii) the stepwise changes in $R^{2}$, and (iii) the standardized regression weights $(\beta)$ for each predictor variable (for the final model). Moreover, significant interaction effects were plotted to facilitate data interpretation. An alpha level of $p<.05$ was determined across all analyses, and all tests were performed with SPSS $®$ (Statistical Package for the Social Sciences; version 24, IBM Corporation, Armonk, NY, USA) for Apple Mac $®$. 


\section{Results}

Descriptive statistics for the main study variables are shown in Table 1. At baseline, 31 participants (12\%) reported clinically relevant burnout symptoms (SMBM $\geq 4.40)$, whereas 23 participants (9\%) exhibited clinically relevant symptoms of depression (PHQ9 >14). At follow up, 27 (14\%) of the athletes reported clinically relevant burnout symptoms, whereas the rate of clinically relevant depressive symptoms dropped to two percent $(n=3)$. More information about the distribution of burnout and depressive symptoms is provided as supplementary online material.

Table 1 further shows that training load and years in competitive sport were not associated with the outcome variables. Table 1S (see supplementary online material) further reveals that females reported more stress and more depressive symptoms, whereas males scored higher on mental toughness. Being currently injured was associated with lower stress levels, higher mental toughness scores, and lower burnout symptoms, whereas athletes with a current injury perceived more depressive symptoms. Similarly, use of medication was associated with more depressive symptoms.

With regard to bivariate correlations, at baseline, higher stress scores were associated with lower mental toughness $(r=-.66, \mathrm{p}<.001)$, but higher burnout levels $(r=.54, p<.001)$ and more depressive symptoms $(r=.29, p<.001)$. By contrast, higher mental toughness was related to lower burnout scores $(r=-.48, p<.001)$ and fewer depressive symptoms $(r=-.30, p<.001)$. Finally, higher burnout scores and higher depressive symptoms were positively associated with each other $(r=.40$, $p<.001)$.

Based on the cross-sectional data, Table 2 supports the notion of mental toughness as a stressbuffer for burnout symptoms, but not depressive symptoms. As shown in Figure 1A, differences in burnout symptoms between participants with low versus high mental toughness scores were small at low levels of perceived stress. By contrast, compared to athletes with high mental toughness, peers with low scores reported more burnout symptoms if they perceived elevated levels of stress. With regard to depressive symptoms, the interaction pointed in the same direction (Figure 1A), but was not statistically significant (Table 2). 
The prospective analyses further underpin the relevance of mental toughness as a stress-buffer. As shown in Table 2, after controlling for confounders, baseline levels of burnout, and main effects, the interaction between stress and mental toughness (assessed at baseline) predicted burnout at followup. Whereas baseline mental toughness was not associated with follow-up burnout scores among athletes at low levels of perceived stress, a scissor effect appeared among athletes who were exposed to high levels of perceived stress (Figure 1B). Thus, among athletes with elevated levels of perceived stress, those who had high mental toughness at baseline, reported fewer burnout symptoms at followup. As illustrated in Figure 1B, a similar pattern of associations was found for depressive symptoms (Table 2).

\section{Discussion}

The following key findings of the present study need special emphasis: First, approximately $10 \%$ of all assessed junior elite athletes reported burnout or depressive symptoms of potential clinical relevance. Second, medium-to-large positive correlations existed between levels of perceived stress and symptoms of mental ill-health. Third, mental toughness buffered some of the negative consequences associated with high levels of perceived stress. This relationship was observed in both the cross-sectional and prospective analyses.

The present study expands upon previous research by showing that beyond main effects, ${ }^{15}$ mental toughness has the capacity to protect young elite athletes from stress-related symptoms of mental ill-health. Our study supports previous findings observed in non-athlete populations, ${ }^{18-20}$ and corroborates the notion of mental toughness as a prominent coping resource among elite athletes. ${ }^{26-28}$ Particular strengths of the present study were that we had access to all students attending Swiss Olympic Sport classes in North-Western Switzerland, and that approximately $90 \%$ of all students participated in our study. Moreover, baseline levels were controlled in the prospective analyses, which provides stronger evidence for causal claims between variables. Finally, we used two instruments with validated cut-off scores to assess clinically relevant levels of burnout and depressive symptoms. These insights are important because based on the Athlete Burnout Questionnaire (ABQ), ${ }^{29}$ researchers have 
estimated the rate of athlete burnout to vary between $1-10 \% .{ }^{13}$ However, as highlighted by Gustafsson et al. ${ }^{13}$ and Isoard-Gautheur et al., ${ }^{30}$ these figures must be interpreted with extreme caution, because the $\mathrm{ABQ}$ is not suitable as a screening measure, and researchers classified athletes as "burned out" based on clinically arbitrary cut-off scores. Referring to internationally accepted standards, our study confirms that a considerable number of athletes shows indication of mental disorders. ${ }^{3,4}$ Researchers have suggested that the incidence of mental health issues might be on the rise due to increasing training loads, competition, and pressure from both inside and outside the elite sport setting. ${ }^{31}$ Consequently, we claim that preventive measures are needed to reduce potential sources of stress, to ensure athletes' well-being, and to minimize the risk of early dropout from elite sport. ${ }^{5}$ With regard to junior elite sports, experts have emphasized that often young elite athletes might not know the psychological or physical symptoms of stress associated with high training loads, ${ }^{9}$ which puts them at a particularly high risk for injury, overtraining or burnout. ${ }^{32}$ While these findings highlight the necessity to sensitize junior athletes for stress-related issues, our study shows that besides other psychological factors such as optimism ${ }^{33}$ and mindfulness, ${ }^{34}$ efforts to promote mental toughness might be a promising avenue to enhance athletes' capacity to cope with stress. While researchers showed that the level of mental toughness depends on genetic factors, ${ }^{35}$ research also showed that the level of mental toughness might change during adolescence, ${ }^{20}$ and may therefore be modifiable through specific interventions.

The present study has a number of limitations. First, only elite athletes from Swiss Olympic Sport Classes of the North-Western part of Switzerland were included. As such, future studies should aim to include a wider range of young elite athletes from different countries to ensure the findings generalize beyond the present sample. Second, although all students had a Swiss Olympic Talents card, the level of "eliteness" might have varied. Nevertheless, 97\% of students had training loads of $\geq 10$ hours per week, showing that their involvement in sport was high. Third, whereas the follow-up period was longer than in previous studies in this area, ${ }^{15}$ it could still be considered "shortitudinal" ${ }^{36}$ Accordingly, future studies should aim to investigate these relationships over longer periods of training to determine if the stress-buffering effects of mental toughness persist. Fourth, although we 
tested interactions, power calculations were based on direct effects. While researchers have highlighted that most prior research assessing moderation effects was underpowered, ${ }^{37}$ interaction effects were generally supported in our study. Finally, we relied on self-reports for data collection. Future research should aim to include experts' ratings on symptoms of depression and burnout to determine if further psychiatric issues might be revealed.

\section{Conclusion}

The present findings suggest that every eighth young elite athlete may present with clinically relevant levels of burnout or depressive symptoms. Furthermore, while high perceived stress was associated with increased psychological health complaints, mental toughness was able to off-set some of the negative consequences resulting from high stress exposure.

\section{Practical implications}

- Instruments with clinical validated cut-offs are recommended to assess burnout and depression in elite athletes.

- From the perspective of positive youth development, helping young elite athletes to develop mental toughness seems to be a valuable undertaking.

- Such an attitude is characterized by not giving up when difficulties arise, seeing problems as an opportunity, feeling able to sort out problems, and having confidence in one's ability to deal constructively with other people.

\section{Acknowledgements}

The project was carried out without external funding. 


\section{References}

1. Mellalieu SD, Neil R, Hanton S et al. Competition stress in sport performers: Stressors experienced in the competition environment. J Sports Sci 2009; 27:729-744.

2. Sabato TM, Walch TJ, Caine DJ. The elite young athlete: strategies to ensure physical and emotional health. Open Access J Sports Med 2016; 7:99-113.

3. Rice SM, Purcell R, De Silva S et al. The mental health of elite athletes: A narrative systematic review. Sports Med 2016; 46:1333-1353.

4. Gulliver A, Griffith KM, Mackinnon A et al. The mental health of Australian elite athletes. $J$ Sci Med Sport 2015; 18:255-261.

5. Isoard-Gautheur S, Guillet-Descas E, Gustafsson H. Athlete burnout and the risk of dropout among young elite handball players. The Sport Psychologist. 2016; 30:123-130.

6. Lazarus RS, Launier R. Stress related transactions between person and environment. In: Pervin L, Lewis M, eds. Perspectives in interactional psychology. New York: Plenum; 1978:287-327.

7. Beckmann J, Ehrlenspiel F. Strategien der Stressregulation im Leistungssport. In: Fuchs R, Gerber M, eds. Stressregulation und Sport. Heidelberg: Springer; 2017.

8. Fletcher D, Sarkar M. Psychological resilience: A review and critique of definitions, concepts, and theory. Psychol Sport Exerc 2012; 18:12-23.

9. Sarkar M, Fletcher D. Psychological resilience in sport performers: A review of stressors and protective factors. J Sports Sci 2014; 32:1419-1434.

10. Luthar SS, Cicchetti D. The construct of resilience: implications for interventions and social policies. Dev Psychopathol. 2000; 12:857-885.

11. Gustafsson H, Kentää G, Hassmén P. Athlete burnout: An integrated model and future research directions. Int Rev Sport Exerc Psychol 2011; 4:3-24.

12. Melamed S, Shirom A, Toker S et al. Burnout and risk of cardiovascular disease: Evidence, possible causal paths, and promising research directions. Psychol Bull. 2006; 132:327-353.

13. Gustafsson H, DeFreese JD, Madigan DJ. Athlete burnout: Review and recommendations. Curr Opin Psychol. 2017; 16:109-113. 
14. Ommundsen Y, Roberts GC, Lemyre P-N et al. Parental and coach support or pressure on psychosocial outcomes of pediatric athletes in soccer. Clin J Sport Med 2006; 16:522-526.

15. Madigan DJ, Nicholls AR. Mental touhgness and burnout in junior athletes: A longitudinal investigation. Psychol Sport Exerc 2017; 32:138-142.

16. Lin $\mathrm{Y}$, Clough PJ, Welch $\mathrm{J}$ et al. Individual differences in mental toughness associate with academic performance and income. Personality and Individual Differences. 2017; 113:178183.

17. Gucciardi DF, Gordon S, Dimmock JA. Advancing mental toughness research and theory using personal construct psychology. Int Rev Sport Exerc Psychol. 2009; 2:54-72.

18. Gerber M, Kalak N, Lemola S et al. Are adolescents with high mental toughness levels more resilient against stress? Stress Health. 2013; 29:164-171.

19. Gerber M, Feldmeth AK, Lang $\mathrm{C}$ et al. The relationship between mental toughness, stress, and burnout among adolescents: A longitudinal study with Swiss vocational students. Psychol Rep $2015 ; 117: 703-723$.

20. Gerber M, Brand S, Feldmeth AK et al. Adolescents with high mental toughness adapt better to perceived stress: A longitudinal study with Swiss vocational students. Pers Individ Dif 2013; 54:808-814.

21. Clough P, Earle K, Sewell D. Mental toughness: The concept and its measurement. In: Cockerill I, ed. Solutions in sport psychology. London: Thomson Learning; 2002:32-46.

22. Cohen S, Kamarck T, Mermelstein R. A global measure of perceived stress. J Health Soc Behav $1983 ; 24: 385-396$.

23. Lerman Y, Melamed S, Shragin Y et al. Association between burnout at work and leukocyte adhesiveness/aggregation. Psychosom Med 1999; 61:828-833.

24. Lundgren-Nilsson A, Jonsdottir IH, Pallant J et al. Internal construct validity of the ShiromMelamed Burnout Questionnaire (SMBQ). BMC Public Health. 2012:doi:10.1186/1471-2458$1112-1181$. 
25. Kroenke K, Spitzer RL, Williams JBW. The PHQ-9. Validity of a brief depression severity measure. J Gen Intern Med 2001; 16:606-613.

26. Kaiseler M, Polman RCJ, Nicholls AR. Mental toughness, stress, stress appraisal, coping and coping effectiveness in sport. Pers Individ Dif 2009; 47:728-733.

27. Nicholls AR, Polman, R.C.J., Levy AR et al. Mental toughness, optimism, pessimism, and coping among athletes. Pers Individ Dif. 2008; 44:1182-1192.

28. Petrie TA, Deiters J, Harmison RJ. Mental toughness, social support, and athletic identity: Moderators of the life stress-injury relationship in collegiate football players. Sport Exerc Performance Psychol. 2014; 3:13-27.

29. Raedeke TD, Smith AL. Development and preliminary validation of an athlete burnout measure. J Sport Exerc Psychcol 2001; 23:281-306.

30. Isoard-Gautheur S, Martinent G, Descas Guillet E et al. Development and evaluation of the psychometric properties of a new measure of athlete burnout: The Athlete Burnout Scale (ABOS). Int J Stress Manag in press: doi:10.1037/str0000083.

31. Gould D, Dieffenbach K, Moffett A. Psychological characteristics and their development in Olympic champions. J Appl Sport Psychol 2002; 14:172-204.

32. Hogan K, Gross R. Overuse injuries in pediatric athletes. Orthop Clin North Am 2003; 34:405415.

33. Gustafsson H, Skoog T. The mediational role of perceived stress in the relation between optimism and burnout in competitive athletes. Anxiety Stress Coping 2012; 25:183-199.

34. Gustafsson H, Davis P, Skoog T et al. Mindfulness and its relationship with perceived stress, affect and burnout in elite junior athletes. J Clin Sport Psychol 2015; 9:263-281.

35. Horsburgh V, Schermer J, Veselka L et al. A behavioural genetic study of mental toughness and personality. Personality and Individual Differences. 2009; 46:100-105.

36. Dormann C, Griffin MA. Optimal time lags in panel studies. Psychol Methods 2015; 20:489.

37. Aguinis H, Edwards JR, Bradley KJ. Improving our understanding of moderation and mediation in strategic management researchq. Organ Res Methods. 2017; 20:665-685. 

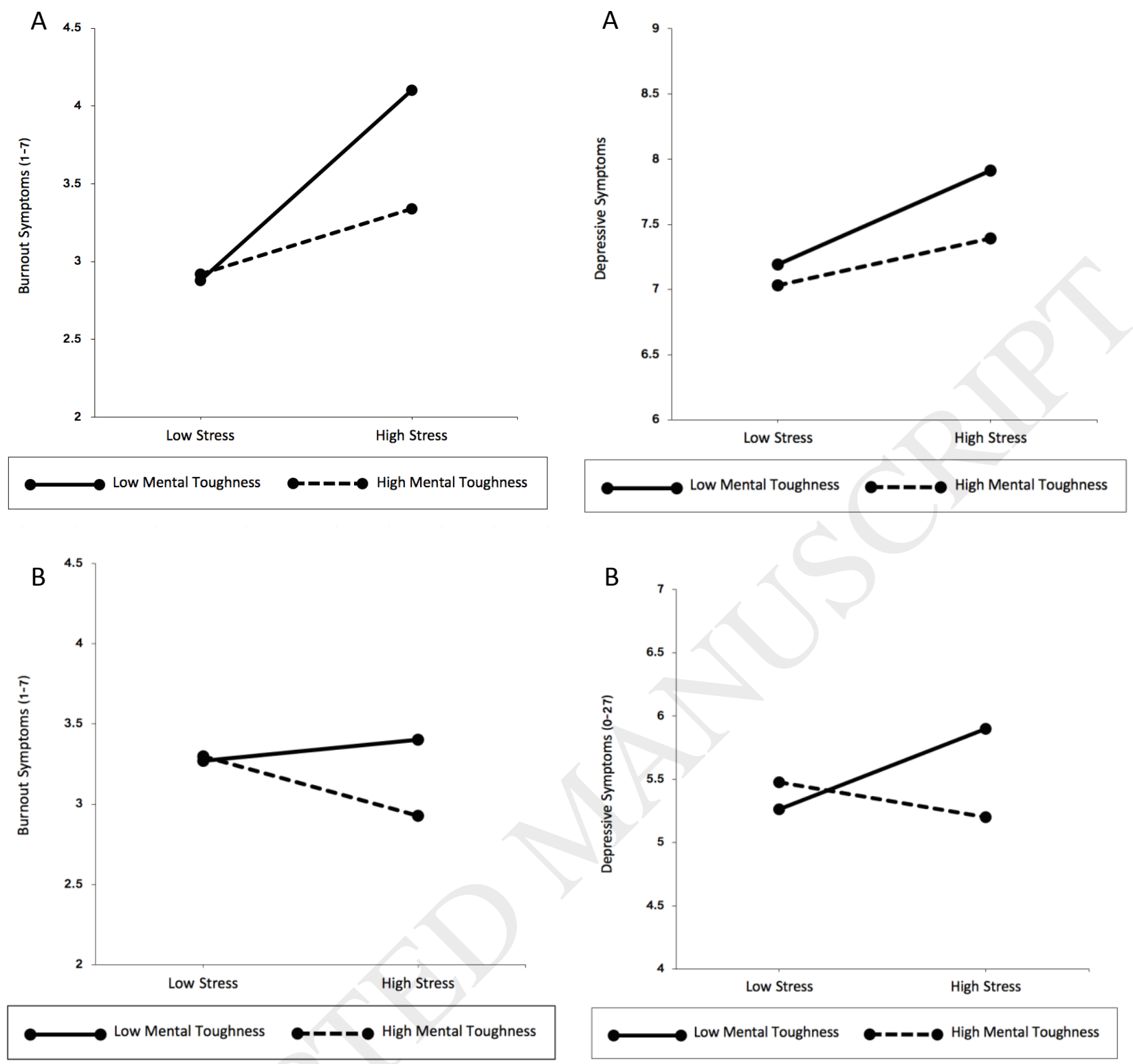

Figure 1. Two-way interactions between perceived stress and mental toughness on burnout symptoms, depressive symptoms and quality of life, based on cross-sectional data $(A)$ and prospective data (B) 
Table 1. Descriptive statistics, psychometric properties of the main study variables, and associations with potential confounders at baseline

\begin{tabular}{|c|c|c|c|c|c|c|c|c|c|c|}
\hline & \multirow[t]{2}{*}{$M$} & \multirow[t]{2}{*}{$S D$} & \multirow[t]{2}{*}{$\alpha$ (items) } & \multicolumn{2}{|l|}{ Range } & \multirow[t]{2}{*}{ Skewness } & \multirow{2}{*}{$\begin{array}{l}\text { Kurtos } \\
\text { is }\end{array}$} & \multicolumn{3}{|c|}{ Correlations with ... } \\
\hline & & & & Potential & Actual & & & ... age & $\begin{array}{l}\text {... training } \\
\text { load }\end{array}$ & $\begin{array}{l}\ldots \text { years in } \\
\text { competitive } \\
\text { sport }\end{array}$ \\
\hline Perceived Stress (PSS) & 2.64 & 0.49 & $.80(10)$ & $1-5$ & $\begin{array}{l}1.00- \\
4.30\end{array}$ & 0.08 & 0.42 & -.02 & .02 & -.03 \\
\hline Mental Toughness (MTQ18) & 3.45 & 0.43 & $.75(18)$ & $1-5$ & $\begin{array}{l}2.00- \\
4.44\end{array}$ & -0.31 & 0.38 & -.02 & -.01 & -.02 \\
\hline Burnout Symptoms (SMBM) & 3.31 & 0.95 & $.92(14)$ & $1-7$ & $\begin{array}{l}1.29- \\
6.79 \\
\end{array}$ & 0.41 & 0.25 & .01 & .01 & .03 \\
\hline $\begin{array}{l}\text { Depressive Symptoms (PHQ- } \\
\text { ) }\end{array}$ & 7.38 & 4.70 & $.85(9)$ & $0-27$ & $0-24$ & 1.04 & 1.06 & .00 & .03 & -.08 \\
\hline
\end{tabular}

Notes. PSS=Perceived Stress Scale. MTQ18=Mental Toughness Questionnaire (18-item short form). SMBM=Shirom-Melamed Burnout Measure. PHQ-9=Patient Health Questionnaire (9-item short form). SWLS=Satisfaction with Life Scale.

$* p<.05$. 
Table 2. Cross-sectional and prospective hierarchical multiple regression analyses predicting burnout symptoms, quality of life and depressive symptoms with perceived stress and mental toughness

\begin{tabular}{|c|c|c|c|c|c|}
\hline & \multicolumn{2}{|c|}{$\begin{array}{l}\text { Cross-sectional analyses } \\
(N=257)\end{array}$} & & \multicolumn{2}{|c|}{$\begin{array}{l}\text { Prospective analyses } \\
(N=197)\end{array}$} \\
\hline & Burnout (T1) & $\begin{array}{l}\text { Depressive } \\
\text { symptoms } \\
\text { (T1) }\end{array}$ & & Burnout (T2) & $\begin{array}{l}\text { Depressive } \\
\text { symptoms } \\
\text { (T2) }\end{array}$ \\
\hline Step $1\left(\Delta R^{2}\right)$ & $.041^{*}$ & $.346 * * *$ & Step $1\left(\Delta R^{2}\right)$ & .011 & .033 \\
\hline Gender $(\beta)$ & .06 & -.06 & Gender $(\beta)$ & .03 & .01 \\
\hline Injury status $(\beta)$ & -.05 & $.50 * * *$ & Injury status $(\beta)$ & .02 & $-.13^{+}$ \\
\hline Medication intake $(\beta)$ & -.01 & $.18^{* *}$ & Medication intake $(\beta)$ & -.09 & $-.15^{*}$ \\
\hline Step $2\left(\Delta R^{2}\right)$ & $.256 * * *$ & $.124 * * *$ & Step $2\left(\Delta R^{2}\right)$ & $.303 * * *$ & $.303^{* * *}$ \\
\hline Perceived stress $(\beta)$ & $.41 * * *$ & $.27 * * *$ & Perceived stress $(\beta)$ & $.50 * * *$ & $.50 * * *$ \\
\hline Step $3\left(\Delta R^{2}\right)$ & $.027^{* *}$ & $.018^{* * *}$ & Step $3\left(\Delta R^{2}\right)$ & .000 & $.016^{*}$ \\
\hline Mental toughness $(\beta)$ & $-.18^{* *}$ & $-.17^{* *}$ & Perceived stress $(\beta)$ & -.06 & $.09 *$ \\
\hline Step $4\left(\Delta R^{2}\right)$ & $.038 * * *$ & $.007^{+}$ & Step $4\left(\Delta R^{2}\right)$ & .008 & $.013^{+}$ \\
\hline $\begin{array}{l}\text { Stress } x \text { mental } \\
\text { toughness }(\beta)\end{array}$ & $-.20 * * *$ & $-.09^{+}$ & Mental toughness $(\beta)$ & -.11 & -.12 \\
\hline \multirow[t]{3}{*}{ Total $R^{2}$} & $.362 * * *$ & $.495^{* * *}$ & Step $5\left(\Delta R^{2}\right)$ & $.014 *$ & $.048^{* * *}$ \\
\hline & & & $\begin{array}{l}\text { Stress } \times \text { mental } \\
\text { toughness }(\beta)\end{array}$ & $-.13^{*}$ & $-.23 * * *$ \\
\hline & & & Total $R^{2}$ & $.337 * * *$ & $.413 * * *$ \\
\hline
\end{tabular}

Notes. Regression weights are presented as they are after step 4 (cross-sectional analyses) or 5 (longitudinal analyses). Across all analyses, baseline values of gender, injury status, medication intake, stress, mental toughness and stress $x$ mental toughness were used as predictors of burnout and depressive symptoms.

${ }^{* * *} p<.001 .{ }^{* *} p<.01 .{ }^{*} p<.05 .{ }^{+} p<.10$ 